\title{
VARIAÇÃO GENÉTICA EM PROGÊNIES DE Myracrodruon urundeuva F.F. \& M.F. ALLEMÃO EM TRÊS SISTEMAS DE CULTIVO ${ }^{1}$
}

\author{
Miguel Luiz Menezes Freitas ${ }^{3}$, Ana Paula de Andrade Aukar², Alexandre Magno Sebbenn ${ }^{3}$, Mario Luiz
} Teixeira de Moraes ${ }^{4}$ e Eliana Gertrudes Macedo Lemos²

\begin{abstract}
RESUMO - Myracrodruon urundeuva (aroeira) é uma das espécies arbóreas que apresentam madeira de maior durabilidade em contato com o solo. Em razão dessa característica, populações naturais de M. urundeuva, distribuídas por quase todo o Brasil, vêm sendo dizimadas. Desse modo, o presente trabalho teve por objetivo avaliar a expressão da variação genética em uma população natural de $M$. urundeuva procedente da Estação Ecológica do Instituto Florestal, localizada em Paulo de Faria, SP. Coletaram-se sementes de 30 árvores de polinização livre, em setembro de 1996. Com esse material, foram instalados três testes de progênies em diferentes sistemas de plantio em Selvíria, MS, obedecendo a um delineamento em blocos casualizados, com 30 tratamentos (progênies) e três repetições. Foi assumido o modelo de cruzamento misto para essa espécie. Verificou-se que houve diferença significativa nos efeitos de progênies e ambiente (testes de progênies), e a interação progênies $\mathrm{x}$ ambientes não foi significativa. Desse modo, as progênies apresentaram comportamento semelhante nos diferentes ambientes. A melhor "performance" das progênies foi no plantio envolvendo $M$. urundeuva, Guazuma ulmifolia (Lam) e Anandenanthera falcata (Benth. Speg.), as quais também apresentaram considerável variação genética, indicando alto valor para programas de conservação e melhoramento genético.
\end{abstract}

Palavras-chave: Myracrodruon urundeuva, variabilidade genética, genética quantitativa e conservação genética.

\section{GENETIC VARIATION IN PROGENIES OF Myracrodruon urundeuva F.F. \& M.F. ALLEMAO IN THREE CULTIVATION SYSTEMS}

\begin{abstract}
Myracrodruon urundeuva is one of the tree species the wood that presents the longest durability when in contact with soil. Because of this characteristic, populations of $\boldsymbol{M}$. urundeuva have been decimated throughout Brazil. Therefore, this work aimed at analyzing the expression of genetic variation in a natural population of M. urundeuva from the Ecological Station of the Forest Institute, located in Paulo de FariaSP. Seeds were collected from 30 free-pollination trees in September, 1996. By using this material, three progeny trials were installed in different planting systems in Selvíria-MS, following a block design with 30 treatments (progenies) and three replications. The mixed crossbreeding model for M. urundeuva was assumed. Significant differences were observed for progeny effects (progeny trials) although the progenies-environments interaction was not significant. Thus, progenies presented the same behavior in different environments; however, their best performance was in the planting involving M. urundeuva, Guazuma ulmifolia (Lam) and Anandenanthera falcata (Benth. Speg.). The progenies also presented considerable genetic variation; hence its utilization in programs for conservation and genetic improvement.
\end{abstract}

Keywords: Myracrodruon urundeuva, genetic variability, genetic quantitative and genetic conservation.

\footnotetext{
${ }^{1}$ Recebido em 13.02.2004 e aceito para publicação em 05.04.2006.

${ }^{2}$ FCAV/UNESP, Via de Acesso Prof. Paulo D. Castellane, s/n 14884-900 Jaboticabal, SP.

${ }^{3}$ Instituto Florestal de São Paulo, Caixa Postal 1322, 01059-970 São Paulo, SP. E-mail: <miguellmfreitas@ yahoo.com.br>.

${ }^{4}$ FEIS/UNESP, Av. Brasil Centro, 56, 15385-000 Ilha Solteira, SP.
} 


\section{INTRODUÇÃO}

Myracrodruon urundeuva (Anacardiaceae), vulgarmente conhecida como aroeira, é uma espécie de ampla ocorrência no Brasil, com exceção da região Amazônica e dos Estados do Rio Grande do Sul e de Santa Catarina, na Região Sul. É uma planta dióica, alógama, cuja incidência em florestas primárias pode ocorrer com diversas espécies, ao contrário das florestas secundárias, onde é de ocorrência quase que homogeneamente, em áreas perturbadas. Em populações naturais, a espécie é comum, porém se encontra em franca erosão em seu "habitat", fazendo parte da lista da FAO de espécies ameaçadas de extinção (FAO, 1986).

O declínio do tamanho populacional e da variabilidade genética entre e dentro dessas populações é caracterizado pela expansão da população humana, devido à utilização de forma extrativista e irracional dos recursos naturais na região de ocorrência de $M$. urundeuva. A conservação dos recursos genéticos, mesmo para aquelas populações que apresentam alta taxa de variabilidade genética e estão fora da lista de espécies ameaçadas de extinção, é fundamental para as futuras gerações, no que diz respeito ao melhoramento da espécie e ao aproveitamento de genes específicos de interesse.

A redução no tamanho das populações pode ser revertida por práticas de manejo de reposição, quando a população for detectada como em vias de extinção ou apresentar níveis altos de endogamia e parentesco e baixo tamanho efetivo populacional (SEBBENN e ETTORI, 2001). Em programas de melhoramento de espécies regionais, vem-se processando, gradativamente, a perda de variabilidade genética devido, principalmente, ao pouco interesse em conservar material que não apresente características desejáveis (PAIVA e VALOIS, 2001).

Estudos fenotípicos e genotípicos entre e dentro de populações, para diferentes características, são as formas mais apropriadas para quantificar a estrutura genética de uma espécie, desde que as sementes colhidas de indivíduos e, ou, populações representativas sejam testadas em condições de laboratório, viveiro ou campo, com o controle dos efeitos ambientais a partir de delineamentos experimentais adequados, como é o caso dos ensaios de progênies ou procedências (KAGEYAMA e DIAS, 1985). Portanto, é possível manter os níveis de variabilidade genética das populações a partir da conservação $e x$ situ da espécie em perigo, que é uma prática recomendável para ampliação e manutenção da variabilidade genética (SEBBENN e ETTORI, 2001).

A utilização de testes de progênies consorciados é um procedimento importante na conservação genética ex situ de espécies arbóreas tropicais, segundo Hamrick (1983) e Kageyama (1990), nas quais é dada ênfase à grande diversidade de espécies na floresta tropical, com sistemas de polinização, predominantemente, via animal e ecossistemas com alta heterogeneidade ambiental. Desse modo, este trabalho teve como objetivo estimar variação genética de uma população de $M$. urundeuva, para fins de conservação e melhoramento genético, utilizando-se de caracteres quantitativos de importância silvicultural, em três sistemas de cultivo.

\section{MATERIAL E MÉTODOS}

Testes de progênies - As sementes de polinização livre de $M$. urundeuva, para os testes de progênies, foram coletadas em 30 árvores (matrizes) na Estação Ecológica do Instituto Florestal (EEPF), localizada em Paulo de Faria, SP, em setembro de 1996. A coleta ocorreu em setembro de 1996, sendo a seleção das matrizes aleatória no campo. Contudo, tomou-se o cuidado de coletar sementes em matrizes espaçadas com uma distância de pelo menos 100 m, a fim de evitar amostrar sementes de árvores parentas. A escolha das matrizes não se deu com base na seleção de nenhum caráter fenotípico (diâmetro, altura, forma etc.). Em cada árvore foi coletado quantidade de frutos suficiente para a obtenção de 100 sementes de cada matriz (aproximadamente 100 frutos/árvore, sendo que cada fruto contém, geralmente, uma semente). AEEPF localizase na região norte do Estado de São Paulo, à margem do Rio Grande (Represa de Água Vermelha), divisa com o Estado de Minas Gerais, região do Triângulo Mineiro. A área que corresponde à Estação Ecológica é de 435,73 ha, entre as coordenadas $19^{\circ} 55^{\prime}$ a $19^{\circ} 58^{\prime}$ de latitude S e 4931' a 49³2' de longitude O. Os solos pertencem à unidade taxonômica Latossolo Roxo. A vegetação foi classificada como floresta mesófila semidecídua, com variações fisionômicas decorrentes de fatores edáficos e sucessionais (STRANGHETTI, 1996). As sementes das outras espécies arbóreas: Guazuma ulmifolia Lam (mutambo) e Anandenanthera falcata Benth. Speg. (angico), que participaram como sombreadoras, foram coletadas na região de Ilha Solteira, $\mathrm{SP}$, e as sementes de Eucalyptus citriodora (Hook) 
foram cedidas pelo Instituto de Pesquisas e Estudos Florestais - IPEF/ESALQ/USP, provenientes de Restinga, SP.

Os experimentos foram conduzidos em condições de campo, na Fazenda de Ensino, Pesquisa e Extensão (FEPE) da Faculdade de Engenharia de Ilha Solteira, da Universidade Estadual Paulista (FEIS/UNESP), no Município de Selvíria, MS, localizada nas coordenadas geográficas de $22^{\circ} 22^{\prime}$ de latitude S e $51^{\circ} 22^{\prime}$ longitude $\mathrm{O}$, com altitude de $335 \mathrm{~m}$. O solo do local, segundo a classificação da Embrapa (1999), é um Latossolo Vermelho Distrófico típico argiloso a moderado, hipidistrófico, álico, caulinítico, férrico, compactado, muito profundo, moderadamente ácido.

Foram instalados três testes de progênies, sendo o primeiro e o segundo em plantios mistos (plantio heterogêneo-AMA: aroeira consorciada com mutambo e angico, AEU: aroeira consorciada com eucalipto), e o terceiro em plantio puro (ARO: somente de aroeira). AMA e ARO foram plantados em março e AEU em maio de 1997.

O delineamento experimental utilizado, em todos os testes, foi o de blocos casualizados. As progênies de $M$. urundeuva foram plantadas no AMA, seguindo o espaçamento de 3,0 x 3,0 m; AEU no espaçamento de 6,0 x 3,0 m; e ARO no espaçamento de 3,0 X 1,5 $\mathrm{m}$. Em todos os ensaios, foram utilizados 30 tratamentos (progênies) e três repetições. As parcelas foram plantadas de forma linear, com 10 plantas nos testes AMA e ARO e com seis plantas no AEU. No AMA, cada planta de M. urundeuva ficou entre uma planta de A. falcata e outra de G. ulmifolia a uma distância de $1,5 \mathrm{~m}$, na mesma linha de plantio, enquanto no AEU M. urundeuva e E. citriodora foram plantados em linhas intercaladas, separadas por $3 \mathrm{~m}$.

Coleta de dados - Os caracteres quantitativos foram avaliados nos testes de progênies, aos três e quatro anos após o plantio. Os caracteres avaliados foram: a) altura total, em metros (ALT); b) diâmetro médio da copa, em metros (DMC); c) forma do fuste (FOR), tendo por base a escala de notas utilizada por Freitas (2003), com amplitude de 1 (altura do fuste até a formação da copa é de no mínimo $50 \mathrm{~cm}$ do solo) a 5 (altura do fuste até a formação da copa ocorre acima de $200 \mathrm{~cm}$ do solo); e d) diâmetro do fuste, com casca, a $30 \mathrm{~cm}$ do solo, em centímetros (D30). O Incremento Médio Anual (IMA), do caráter altura total da planta, foi calculado para cada ano, dividindo o crescimento, em altura $(\mathrm{m})$, pela idade das árvores.

Análises estatísticas e parâmetros genéticos - $\mathrm{O}$ modelo matemático adotado para as análises individuais, dentro de cada um dos experimentos e tomando-se o efeito de progênies como aleatório, foi: $Y_{i j k}=m+r_{j}+f_{i}+d_{k(i j)}+e_{i j(k)}$

em que: $\mathrm{Y}_{\mathrm{ijk}}$ é a observação na árvore k, da progênie i, na repetição j; $m$ é a média geral; $r_{j}$ é o efeito da repetição $\mathrm{j}$, sendo $\mathrm{j}=1,2, \ldots, \mathrm{r}$; $\mathrm{f}_{\mathrm{i}}$ é o efeito da progênie $\mathrm{i}$, sendo $\mathrm{i}=1,2, \ldots, \mathrm{f} ; \mathrm{d}_{\mathrm{k}(\mathrm{ij})}$ é o desvio referente à árvore $\mathrm{k}$ na parcela $\mathrm{ij}$, com $\mathrm{k}=1,2, \ldots, \mathrm{d} ; \mathrm{e} \mathrm{e}_{\mathrm{ij}(\mathrm{k})}$ é o efeito do erro experimental.

As fontes de variação e as esperanças dos quadrados médios referentes às análises individuais são apresentadas na Tabela 1 .

O modelo matemático adotado na análise conjunta, tomando-se o efeito de experimento como fixo e o de progênies como aleatório, foi:

$$
\mathrm{Y}_{\mathrm{ijkl}}=\mathrm{m}+\mathrm{r}_{\mathrm{j}(\mathrm{k})}+\mathrm{p}_{\mathrm{k}}+\mathrm{f}_{\mathrm{i}(\mathrm{k})}+\mathrm{e}_{\mathrm{ij}(\mathrm{k})}+\mathrm{d}_{\mathrm{l}(\mathrm{ijk})}
$$

em que: $\mathrm{Y}_{\mathrm{ijk}}$ é a observação na árvore 1 , no experimento $\mathrm{k}$, na progênie $\mathrm{i}$, da repetição $\mathrm{j}$; $\mathrm{m}$ é a média geral; $\mathrm{r}_{\mathrm{j}(\mathrm{k})}$ é o efeito da repetição $\mathrm{j}$, no experimento $\mathrm{k}$, em que $\mathrm{j}=$ $1,2, \ldots, r ; p_{k}$ é o efeito do experimento $\mathrm{k}$, sendo $\mathrm{k}=1$, $2, \ldots, p ; f_{i(k)}$ é o efeito da progênie $\mathrm{i}$, no experimento $\mathrm{k}$, em que $\mathrm{i}=1,2, \ldots, \mathrm{f} ; \mathrm{e}_{\mathrm{ij}(\mathrm{k})}$ é o efeito do erro referente à parcela ij, no experimento $\mathrm{k}$; e $\mathrm{d}_{\mathrm{l}(\mathrm{j} \mathrm{jk})}$ é o desvio referente à árvore 1 na parcela $\mathrm{ij}$, no experimento $\mathrm{k}$.

O esquema da análise de variância conjunta, envolvendo os diferentes experimentos, para cada um dos caracteres silviculturais, é apresentado na Tabela 2.

Tabela 1 - Esquema da análise de variância, em nível de média, de parcelas em progênies de $M$. urundeuva

Table 1-Scheme of analyis of variance, at mean level, of M. urundeuva progeny plots

\begin{tabular}{lcccc}
\hline \multicolumn{1}{c}{ FV } & GL & QM & $\mathrm{E}(\mathrm{QM})$ & $\mathrm{F}$ \\
\hline Repetições & $r-1$ & $Q_{1}$ & $(1 / \overline{\mathrm{n}}) \sigma_{\mathrm{d}}^{2}+\sigma_{\mathrm{e}}^{2}+\mathrm{p \sigma}_{\mathrm{b}}^{2}$ & $Q_{1} / Q_{3}$ \\
Progênies & $p-1$ & $Q_{2}$ & $(1 / \overline{\mathrm{n}}) \sigma_{\mathrm{d}}^{2}+\sigma_{\mathrm{e}}^{2}+\mathrm{r} \sigma_{\mathrm{p}}^{2}$ & $Q_{2} / Q_{3}$ \\
Erro & $(r-1)(p-1)$ & $Q_{3}$ & $(1 / \overline{\mathrm{n}}) \sigma_{\mathrm{d}}^{2}+\sigma_{\mathrm{e}}^{2}$ & - \\
\hline Dentro & $r p(n-1)$ & $Q_{4}$ & $\sigma_{\mathrm{d}}^{2}$ & - \\
\hline
\end{tabular}

FV: fonte de variação; GL: graus de liberdade; QM: quadrados médios; $\mathrm{E}(\mathrm{QM})$ : esperança dos quadrados médios; F: Teste-F; r, p e n são, respectivamente, números de repetições de progênies e de plantas dentro de parcelas.

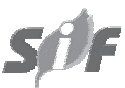

R. Árvore, Viçosa-MG, v.30, n.3, p.319-329, 2006 
Tabela 2 - Esquema da análise de variância conjunta de experimentos, ano nível de média de progênies, em M. urundeuva Table 2 -Scheme of experiment group analysis of variance, at progeny mean level, of $\boldsymbol{M}$. urundeuva progeny plots

\begin{tabular}{lcccc}
\hline \multicolumn{1}{c}{ FV } & GL & QM & $\mathrm{E}(\mathrm{QM})$ & $\mathrm{F}^{1}$ \\
\hline Rep (Exp) & $s(r-1)$ & $Q_{1}$ & $(1 / \bar{n}) \sigma_{d}^{2}+\sigma_{e}^{2}+p \sigma_{R}^{2}$ & $Q_{1} / Q_{5}$ \\
$\operatorname{Exp}(\mathrm{S})$ & $s-1$ & $Q_{2}$ & $(1 / \bar{n}) \sigma_{d}^{2}+\sigma_{e}^{2}+p \sigma_{R}^{2}+r\left(\frac{s}{s-1}\right) p \sigma_{P S}^{2}+r p V_{S}$ & $\frac{\left(Q_{2}+Q_{5}\right)}{\left(Q_{1}+Q_{4}\right)}$ \\
$\operatorname{Prog}(\mathrm{P})$ & $p-1$ & $Q_{3}$ & $(1 / \bar{n}) \sigma_{d}^{2}+\sigma_{e}^{2}+r s \sigma_{P}^{2}$ & $Q_{3} / Q_{5}$ \\
$\operatorname{PxS}$ & $(p-1)(s-1)$ & $Q_{4}$ & $(1 / \bar{n}) \sigma_{d}^{2}+\sigma_{e}^{2}+r\left(\frac{s}{s-1}\right) p \sigma_{P S}^{2}$ & $Q_{4} / Q_{5}$ \\
Erro entre & $s(r-1)(p-1)$ & $Q_{5}$ & $(1 / \bar{n}) \sigma_{d}^{2}+\sigma_{e}^{2}$ & - \\
\hline Erro dentro & $r p s(n-1)$ & $Q_{6}$ & $\sigma_{d}^{2}$ & \\
\hline
\end{tabular}

$\sigma_{P S}^{2}=$ variância da interação progênies por experimento. $\sigma_{P}^{2}=$ variância genética entre progênies. $\sigma_{e}^{2}=$ variância devida ao erro entre parcelas. $\sigma_{d}^{2}=$ variância fenotípica dentro das parcelas. $\bar{n}=$ média harmônica do número de plantas por parcela. $p=$ número de progênies. $s=$ número de experimentos; e $r=$ número de repetições.

A partir das análises de variância, para cada um dos caracteres silviculturais analisados, em nível de média, foram obtidas as estimativas dos parâmetros genéticos e estatísticos, baseando-se na metodologia proposta por Vencovsky e Barriga (1992):

$$
\begin{aligned}
& C V=\frac{100 \cdot \sqrt{Q_{3}}}{\hat{m}} ; C V_{D}=\frac{100 \cdot \sqrt{\hat{\sigma}_{d}^{2}}}{\hat{m}} ; C V_{g}=\frac{100 \cdot \sqrt{\hat{\sigma}_{P}^{2}}}{\hat{m}} ; \\
& C V_{F}=\frac{100 \cdot \sqrt{\hat{\sigma}_{F}^{2}}}{\hat{m}} ; C V_{\bar{F}}=\frac{100 \cdot \sqrt{\hat{\sigma}_{\bar{F}}^{2}}}{\hat{m}} ; \hat{h}^{2}=\frac{\hat{\sigma}_{A}^{2}}{\hat{\sigma}_{F}^{2}} ; \\
& \hat{h}_{(S M)}^{2}=\frac{\hat{r}_{x y} \cdot \hat{\sigma}_{A}^{2}}{\hat{\sigma}_{F}^{2}} ; \hat{h}_{m}^{2}=\frac{Q_{2}-Q_{3}}{Q_{2}} ; \hat{h}_{D}^{2}=\frac{(3 / 4) \cdot \hat{\sigma}_{A}^{2}}{\hat{\sigma}_{d}^{2}} ; \\
& \hat{h}_{D(S M)}^{2}=\frac{\left(1-\hat{r}_{x y}\right) \cdot \hat{\sigma}_{A}^{2}}{\hat{\sigma}_{d}^{2}} \text { e } \hat{r}_{x y}=0,25\left(4 \cdot \hat{s}+\hat{t}^{2}\right)
\end{aligned}
$$

em que: $C V=$ coeficiente de variação experimental; $C V_{D}$ = coeficiente de variação dentro de parcelas; $C V_{G}=$ coeficiente de variação genética; $C V_{F}=$ coeficiente de variação fenotípica; $C V_{\bar{F}}=$ coeficiente de variação fenotípica média; $\hat{h}_{D}^{2}=$ coeficiente de herdabilidade, em nível de plantas dentro de parcelas; $\hat{\mathrm{h}}_{\mathrm{m}}^{2}=$ coeficiente de herdabilidade, em nível de média de parcelas; $\hat{\mathrm{h}}^{2}=$ coeficiente de herdabilidade, em nível de plantas; e $\hat{r}_{x y}=$ coeficiente de parentesco obtido por Freitas (2003) com base em Ritland (1989).
O teste de Tukey, para o efeito de experimentos, foi calculado utilizando o pacote estatístico SAS (1999) e com base em Pimentel-Gomes e Garcia (2002).

\section{RESULTADOS E DISCUSSÃO}

\section{Análises de variância}

Os resultados obtidos no terceiro ano após o plantio (Tabela 3), para o coeficiente de variação experimental (CV), foram baixos para todos os caracteres, nos três ensaios, variando de 3,4 a 13,1\%. Comparando os experimentos, observou-se que o coeficiente de variação experimental foi inferior para todos os caracteres no AMA, seguido do AEU e do ARO. No quarto ano após o plantio, o coeficiente de variação experimental variou de 9,7 a $18,7 \%$, entre os caracteres estudados, com média geral de $14,0 \%$. Comparando os experimentos, observou-se que, assim como no terceiro ano após o plantio, todos os caracteres, com exceção do caráter forma do fuste, o coeficiente de variação experimental foi, em geral, menor no AMA, invertendo-se a seqüência, nesse ano, para o ARO em plantio homogêneo e para o AEU.

Os valores obtidos para o coeficiente de variação experimental (CV), durante os dois anos de coleta de informações, entre os experimentos (Tabela 3), evidenciaram que o experimento AMA apresenta maior controle experimental, devido à interação entre as plantas utilizadas nesse plantio consorciado, as quais representam um modelo de sucessão ecológica, 
aumentando a precisão das estimativas. No entanto, o experimento AEU apresentou-se como intermediário na avaliação do coeficiente de variação experimental para o terceiro ano após o plantio, porém, no quarto ano, esse quadro foi revertido para o ARO. Outro fato observado quanto ao relacionamento entre as espécies refere-se ao ganho de crescimento, em que a quase totalidade dos caracteres aponta para o experimento AMA, entre os dois anos de estudo, com as maiores médias. Valores de coeficiente de variação experimental na ordem de 10 a $20 \%$ podem ser considerados baixos para experimentos onde ocorre competição (PIMENTELGOMES e GARCIA, 2002). Assim, os resultados obtidos indicam que o controle experimental foi satisfatório, e pode-se esperar boa precisão nas estimativas de parâmetros genéticos.

A variabilidade entre as progênies quanto aos caracteres silviculturais, no terceiro ano após o plantio, pode ser verificada nos resultados obtidos para o Teste $F$ nas análises de variância. Não ocorreu diferença significativa entre progênies para os caracteres, com exceção de $\operatorname{ALT}(\mathrm{P}<0,05)$ e D30 ( $<<0,01)$, no teste ARO. No quarto ano após o plantio, observou-se diferença significativa entre progênies para os caracteres ALT $(\mathrm{P}<0,05)$, FOR $(\mathrm{P}<0,05)$ e D30 $(\mathrm{P}<0,05)$, no ARO. Nos demais experimentos, não foram detectadas diferenças significativas.

Verificou-se que, para os caracteres silviculturais, as progênies apresentaram pouca variação genética até o terceiro ano de idade. Tal fato se repetiu no quarto ano, porém com as progênies não expressando variação genética nos ensaios AMA e AEU. Entretanto, a combinação de espécies no experimento AMA resultou em melhor desempenho para os caracteres de crescimento, no terceiro e quarto anos após o plantio (ALT, D30 e DMC), isto é, em plantio combinado, a média dos caracteres foi mais alta, refletindo estes o desenvolvimento da espécie. O plantio combinado (AMA), apesar de ter sua área mais efetivamente ocupada, por incluir outras espécies no mesmo ambiente, fornece, aparentemente, condições melhores para o desenvolvimento e, sobretudo, adaptação da espécie. Considerando que o material genético incluído nos três ensaios é o mesmo, a ausência de diferenças significativas entre progênies, nos experimentos AMA e AEU, indica que as combinações de espécies suprimiram parte da competição entre progênies. Por não compartilhar de ambiente propício, o material plantado em povoamento puro (ARO) foi induzido à competição, refletindo em padrões diferenciados de expressão fenotípica entre progênies. O monitoramento dos caracteres nos experimentos permitiu identificar se a competição em plantios combinados ou puros ocorre mais tardiamente ou não.

No caráter altura da planta, o valor médio de 5,27 $\mathrm{m}$ alcançado pelas progênies, no quarto ano após o plantio, demonstra que houve incremento médio anual (IMA) de 1,32 m, inferior ao aumento de 1,58 m obtido aos três anos de idade.

Tabela 3 - Estimativas da média geral, do teste F (progênies) e do coeficiente de variação experimental (CV), em três testes de progênies: $\mathrm{AMA}^{1}, \mathrm{AEU}^{2} \mathrm{e} \mathrm{ARO}^{3}$, nos caracteres ALT: altura total; FOR: forma do fuste; D30: diâmetro do fuste, com casca, a $30 \mathrm{~cm}$ do solo; e DMC: diâmetro médio da copa, em uma população de M. urundeuva no terceiro e quarto anos de idade, em Selvíria, MS

Table 3 - Estimates of overall mean, F-test (progênies) and experimental coefficient of variation (CV) of three progeny tests: $\mathrm{AMA}^{1}, \mathrm{AEU}^{2}$ and $\mathrm{ARO}^{3}$, for the traits total height (ALT); stem form; D30: stem diameter, with bark, at $30 \mathrm{~cm}$ from the ground; and DMC: average crown diameter, of a $\boldsymbol{M}$. urundeuva population of three and four years of age, in Selvíria, $M S$

\begin{tabular}{|c|c|c|c|c|c|c|c|c|c|c|c|c|c|c|c|c|c|c|}
\hline \multirow{3}{*}{ Caráter } & \multicolumn{6}{|c|}{ AMA } & \multicolumn{6}{|c|}{$\mathrm{AEU}$} & \multicolumn{6}{|c|}{ ARO } \\
\hline & \multicolumn{2}{|c|}{ Média } & \multicolumn{2}{|c|}{$\mathrm{F}$} & \multicolumn{2}{|c|}{$\mathrm{CV}(\%)$} & \multicolumn{2}{|c|}{ Média } & \multicolumn{2}{|c|}{$\mathrm{F}$} & \multicolumn{2}{|c|}{ CV (\%) } & \multicolumn{2}{|c|}{ Média } & \multicolumn{2}{|l|}{$\mathrm{F}$} & \multicolumn{2}{|c|}{$\mathrm{CV}(\%)$} \\
\hline & $3^{\circ}$ & $4^{\circ}$ & $3^{\circ}$ & $4^{\circ}$ & $3^{\circ}$ & $4^{\circ}$ & $3^{\circ}$ & $4^{\circ}$ & $3^{\circ}$ & $4^{\circ}$ & $3^{\circ}$ & $4^{\circ}$ & $3^{\circ}$ & $4^{\circ}$ & $3^{\circ}$ & $4^{\circ}$ & $3^{\circ}$ & $4^{\circ}$ \\
\hline LT & 5,21 & 5,93 & 0,95 & 0,76 & 3,9 & 10,7 & 4,67 & 5,06 & 1,30 & 1,39 & 5,8 & 13,4 & 4,32 & 4,83 & $1,85^{*}$ & $1,52^{*}$ & 6,1 & 12,9 \\
\hline $\mathrm{R}$ & 1,48 & 2,17 & 1,00 & 1,48 & 6,7 & 17,9 & 1,60 & 2,06 & 1,43 & 1,37 & 8,5 & 14 & 05 & 2,46 & 1,69 & $1,23^{*}$ & 13,1 & 18,7 \\
\hline 30( & 6,32 & 7,31 & 1,03 & 0,96 & 3,4 & 10,5 & 4,64 & 5,30 & 1,40 & 1,16 & 5,1 & 16,6 & 5,02 & 5,51 & $2,48 * *$ & $1,66^{*}$ & 6,1 & 14,6 \\
\hline DMC (m) & 2,19 & 2,64 & 1,16 & 1,33 & 4,6 & 9,7 & 1,58 & 2,01 & 0,80 & 1,00 & 6,5 & 16,3 & 2,11 & 2,76 & 1,62 & 1,23 & 7,5 & 12,4 \\
\hline
\end{tabular}

${ }^{1} \mathrm{AMA}$ : aroeira, mutambo e angico; ${ }^{2} \mathrm{AEU}$ : aroeira e eucalipto; ${ }^{3} \mathrm{ARO}$ : só aroeira; * e **: estimativas do teste $\mathrm{F}$ significativas em níveis de 5 e $1 \%$ de significância, respectivamente. 
O IMA diminuiu com o passar dos anos. Freitas et al. (2002) observaram uma desuniformidade de crescimento, em diferentes sistemas de cultivo, em uma população de $M$. urundeuva procedente de Petrolina (PE), em que a planta apresentava, em um ano, crescimento rasteiro, e, em anos posteriores, ocorria uma verticalização de seu tronco, sendo que o inverso também poderia ocorrer. Essa desuniformidade de crescimento, provavelmente, pode ser causada pelos diferentes espaçamentos e sistemas de plantio, e pode provocar grandes diferenças de resultados entre a fase inicial de desenvolvimento da planta até sua estabilização.

Pela análise conjunta entre os experimentos, no terceiro ano após o plantio (Tabela 4), nas progênies e suas possíveis interações, pôde-se observar que, para o caráter ALT, houve diferença significativa em nível de $1 \%$ de probabilidade entre os experimentos e 5\% entre as progênies. Quanto aos demais caracteres, quando ocorreu diferença significativa, essa diferença foi em nível de $1 \%$ de probabilidade, como na FOR e DMC, com diferença significativa somente entre os experimentos; em relação ao D30, houve diferença significativa para os efeitos de experimento e progênie.

No quarto ano após o plantio, pôde-se observar que, entre as progênies, os caracteres ALT e D30 apresentaram diferença significativa em nível de $1 \%$ de probabilidade. Entre os experimentos, todos os caracteres estudados apresentaram diferença significativa em nível de $1 \%$ de probabilidade. Como no primeiro ano de coleta de dados, os resultados no segundo ano realçam a possibilidade de ganhos de seleção entre progênies quando se utilizaram esses caracteres. No terceiro e quarto anos após o plantio, em todos os caracteres estudados não ocorreu diferença significativa para a interação entre experimentos x progênies.

Pelo teste de Tukey, os resultados, no terceiro ano após o plantio (Tabela 5), apresentaram diferença significativa entre os experimentos para todos os caracteres. As maiores médias de ALT, D30 e DMC foram obtidas no experimento AMA, com média de $5,21 \mathrm{~m}, 6,32 \mathrm{~cm}$ e 2,19 m, respectivamente. ALT e D30 destacaram-se no AMA, pois se diferenciaram significativamente dos outros testes de progênies. Para o caráter FOR, o teste de progênie de $M$. urundeuva em plantio homogêneo (ARO) diferenciou-se significativamente dos demais, com média de 2,05. Quanto ao caráter DMC, os testes de progênies de $M$. urundeuva em plantio homogêneo ARO e consorciado AMA diferenciaram-se significativamente do teste AEU, com média de 2,19 e 2,11 m para os melhores testes, respectivamente.

Pelo teste de Tukey, no quarto ano após o plantio (Tabela 5) os experimentos apresentaram diferença significativa para todos os caracteres estudados. Para os caracteres ALT e D30, as melhores médias, de maneira semelhante ao ano anterior, foram obtidas pelo teste de progênies de $M$. urundeuva cultivada em plantio misto (AMA), com média de 5,93 m e 7,31 cm, respectivamente. Para os caracteres forma do fuste e diâmetro médio da copa, o teste de progênie de $M$. urundeuva em plantio homogêneo (ARO) diferenciouse significativamente dos demais, com médias de 2,46 e $2,76 \mathrm{~m}$, respectivamente.

Tabela 4 - Estimativas do teste F, obtidas nas análises de variância conjunta, para altura (ALT), forma do fuste (FOR), diâmetro a $30 \mathrm{~cm}$ (D30) e diâmetro médio da copa (DMC), em uma população de M. urundeuva, no terceiro e quarto anos de idade, em Selvíria, MS

Table 4 -F-test estimates obtained from the group analysis of variance for height (ALT); stem form (FOR); stem diameter at $30 \mathrm{~cm}$ (D30); and average crown diameter (DMC):, of a M. urundeuva population of three and four years of age, in Selvíria, $M S$

\begin{tabular}{|c|c|c|c|c|c|c|c|c|}
\hline \multirow{3}{*}{ Fonte de Variação } & \multicolumn{8}{|c|}{ Caracteres } \\
\hline & \multicolumn{2}{|c|}{ ALT $(\mathrm{m})$} & \multicolumn{2}{|c|}{ FOR } & \multicolumn{2}{|c|}{ D30 $(\mathrm{cm})$} & \multicolumn{2}{|c|}{$\mathrm{DMC}(\mathrm{m})$} \\
\hline & $3^{\circ}$ & $4^{\circ}$ & $3^{\circ}$ & $4^{\circ}$ & $3^{\circ}$ & $4^{\circ}$ & $3^{\circ}$ & $4^{\circ}$ \\
\hline $\operatorname{Exp}(S)^{4}$ & $36,67^{* *}$ & $63,21^{* *}$ & $37,11^{* *}$ & $22,85^{* *}$ & $144,51^{* *}$ & $148,86^{* *}$ & $90,70^{* *}$ & $138,50^{* *}$ \\
\hline $\operatorname{Prog}(\mathrm{P})^{5}$ & $2,08^{*}$ & $2,03^{* *}$ & 1,00 & 1,66 & $2,52^{* *}$ & $2,29^{* *}$ & 1,52 & 1,22 \\
\hline PxS & 0,97 & 0,87 & 0,9 & 1,18 & 1,21 & 0,78 & 1,09 & 1,12 \\
\hline
\end{tabular}

${ }^{4}$ Experimentos; ${ }^{5}$ progênies; $*$ e $* *$ estimativas do teste F significativas em níveis de 5 e $1 \%$ de significância, respectivamente.

R. Árvore, Viçosa-MG, v.30, n.3, p.319-329, 2006 
Tabela 5 - Comparação entre os experimentos ${ }^{1} \mathrm{AMA},{ }^{2} \mathrm{AEU}$ e ${ }^{3} \mathrm{ARO}$, na análise conjunta, para os caracteres altura (ALT), forma do fuste (FOR), diâmetro a $30 \mathrm{~cm}$ (D30) e diâmetro médio da copa (DMC), no terceiro e quarto anos de idade, em Selvíria, MS

Table 5 - Comparison among the experiments ${ }^{1} A M A,{ }^{2} A E U$ and ${ }^{3} A R O$, in the the group analysis for the traits height (ALT); stem form (FOR); stem diameter at $30 \mathrm{~cm}$ (D30); and average crown diameter (DMC):, of a M. urundeuva population of three and four years of age, in Selvíria, $M S$

\begin{tabular}{|c|c|c|c|c|c|c|c|c|}
\hline \multirow{3}{*}{ Fonte de Variação } & \multicolumn{8}{|c|}{ Caracteres } \\
\hline & \multicolumn{2}{|c|}{$\operatorname{ALT}(\mathrm{m})$} & \multicolumn{2}{|c|}{ FOR } & \multicolumn{2}{|c|}{ D30 $(\mathrm{cm})$} & \multicolumn{2}{|c|}{$\mathrm{DMC}(\mathrm{m})$} \\
\hline & $3^{\circ}$ & $4^{\circ}$ & $3^{\circ}$ & $4^{\circ}$ & $3^{\circ}$ & $4^{\circ}$ & $3^{\circ}$ & $4^{\circ}$ \\
\hline AMA & $5,21 \mathrm{~A}$ & $5,93 \mathrm{~A}$ & $1,48 \mathrm{~B}$ & $2,17 \mathrm{~B}$ & $6,32 \mathrm{~A}$ & $7,31 \mathrm{~A}$ & $2,19 \mathrm{~A}$ & $2,64 \mathrm{~B}$ \\
\hline AEU & $4,67 \mathrm{~B}$ & $5,06 \mathrm{~B}$ & $1,60 \mathrm{~B}$ & $2,06 \mathrm{~B}$ & $4,64 \mathrm{C}$ & $5,30 \mathrm{~B}$ & $1,58 \mathrm{~B}$ & $2,01 \mathrm{C}$ \\
\hline ARO & $4,32 \mathrm{C}$ & $4,83 \mathrm{~B}$ & $2,05 \mathrm{~A}$ & $2,46 \mathrm{~A}$ & $5,02 \mathrm{~B}$ & $5,51 \mathrm{~B}$ & $2,11 \mathrm{~A}$ & $2,76 \mathrm{~A}$ \\
\hline
\end{tabular}

Médias seguidas pelas mesmas letras não diferenciaram em nível de $5 \%$ de probabilidade, pelo teste de Tukey. ${ }^{1} \mathrm{AMA}$ : aroeira, mutambo e angico; ${ }^{2} \mathrm{AEU}$ : aroeira e eucalipto; e ${ }^{3} \mathrm{ARO}$ : só aroeira.

O fato de não se ter detectado diferença significativa para a interação entre experimentos e progênies sinaliza para o entendimento da ocorrência de comportamento semelhante dos genótipos em todos os ambientes, conforme é citado por Allard (1971). Porém, há de ressaltarse que o arranjo formado no experimento AMA apresentou as maiores medidas de crescimento das progênies, fortalecendo a hipótese da necessidade de respeitar-se, quando do cultivo, os modelos sucessionais ecológicos. Resultados obtidos por Oliveira et al. (2000) indicaram que o plantio heterogêneo de $M$. urundeuva é promissor para a maioria dos caracteres ligados ao crescimento da planta.

\section{Coeficientes de variação genética}

O valor médio obtido no terceiro ano após o plantio para os caracteres estudados para o coeficiente de variação genética $(\mathrm{CVg})$ foi de 5,7\%. Entre os caracteres, ALT apresentou a maior média, 6,6\%. Para o quarto ano, o valor médio obtido nos caracteres estudados para o coeficiente de variação genética foi de 5,9\%, sendo o maior valor observado em FOR, com 7,7\%.

Observa-se, respectivamente ao terceiro e quarto anos após o plantio (Tabela 6), que os controles genéticos dos caracteres estudados, em média geral, foram baixos. Esse fato está ligado à juvenilidade da planta, pois, geralmente nessa espécie, o amadurecimento - passagem da fase de madeira juvenil a madeira adulta - ocorre em uma idade mais avançada, sendo que, na etapa de desenvolvimento em que $M$. urundeuva se encontra, ainda não alcançou suficiente desenvolvimento para expressar todo o seu potencial genético. Kageyama (1980) observou que, apesar de existirem restrições para estimativas de parâmetros genéticos em idades precoces, em espécies arbóreas, muitas avaliações ocorrem em ensaios jovens, sendo necessário, sempre que possível, a sequiência do acompanhamento dos dados em idades adultas, para garantir maior segurança nas estimativas. Esse fato, também, foi observado por Siqueira et al. (1993; 2000) e Sebbenn et al. (1998).

Comparando-se com a literatura, o valor médio desse experimento corresponde aos obtidos por outros ensaios. Especificamente para ALT, para a qual a maioria dos trabalhos vem sendo direcionadas, verifica-se que, para M. urundeuva, Moraes et al. (1992) obtiveram valores de coeficiente de variação genético inferiores para duas populações, de 3,3 e $3,1 \%$, respectivamente. Oliveira et al. (2000) obtiveram valores médios para o mesmo caráter de $6,45 \%$ entre o período de 7 e 24 meses para plantio heterogêneo e valores nulos para o plantio homogêneo. Sebbenn e Ettori (2001) obtiveram coeficiente de variação genética de $11,3 \%$ para altura total de planta em ensaio misto aos oito anos de idade. Esses resultados evidenciam que o coeficiente de variação genética, obtido nos três testes de progênies, é semelhante aos dados verificados na literatura para a espécie.

No terceiro ano após o plantio, nos dados obtidos para quociente $\hat{b}(\mathrm{CVg} / \mathrm{CV})$, verifica-se que a variação entre os caracteres analisados foi de 0,02 a 0,94, respectivamente, para os caracteres FOR e D30, com uma média geral de 0,46 (Tabela 6). No quarto ano após o plantio, a variação entre os caracteres analisados para o quociente $\hat{b}(\mathrm{CVg} / \mathrm{CV})$ foi de 0,29 a 0,73 , respectivamente, para os caracteres DMC e FOR, com uma média geral de 0,53. Entretanto, o quociente $\hat{b}$ para ALT apresentou, como no ano anterior, média entre os experimentos maior que os demais caracteres. 


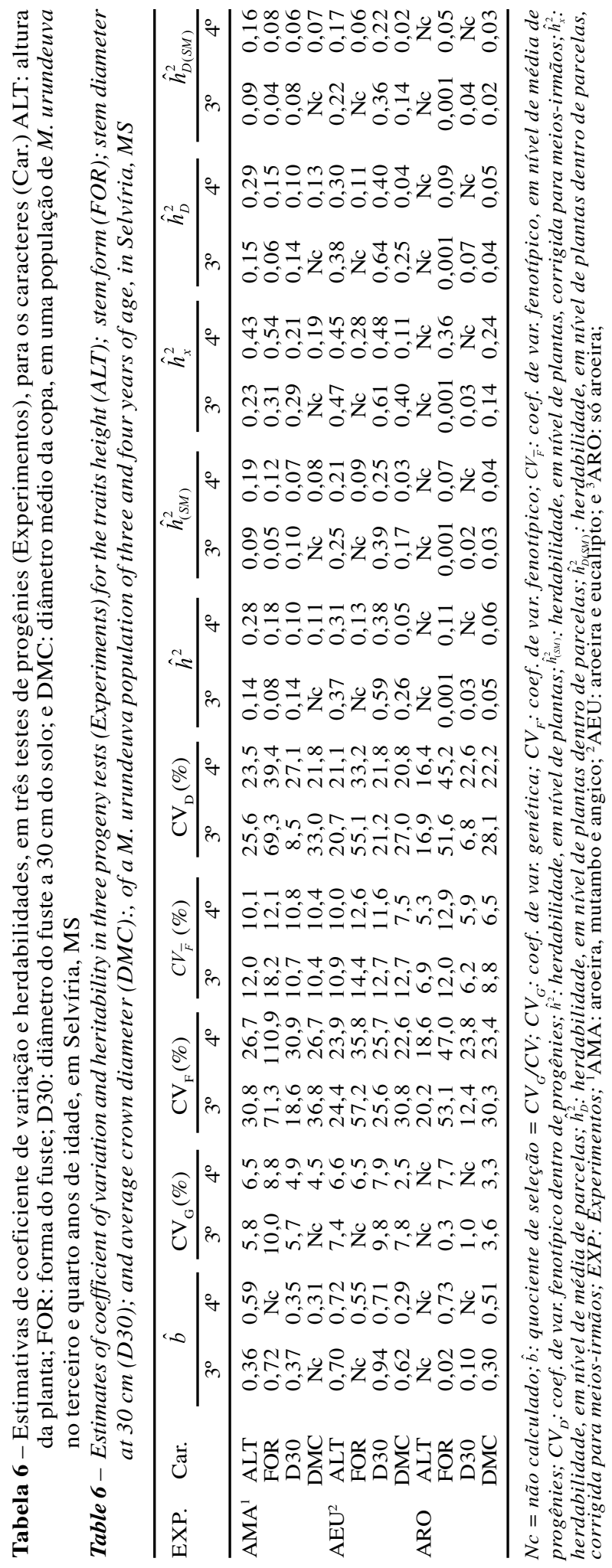

Para o quociente $\hat{b}(\mathrm{CVg} / \mathrm{CV})$, quanto mais próximo de 1,00 (um), melhor o caráter para seleção de progênies (VENCOVSKY, 1987). Se o estudo fosse dirigido ao melhoramento genético da espécie, o principal caráter a ser selecionado seria ALT para os dois anos de coleta de dados. Oliveira et al. (2000) obtiveram baixos valores para o experimento em cultivo heterogêneo (AMA) para o caráter DMC; em contrapartida, FOR apresentou o maior quociente entre o primeiro e o segundo ano de plantio, em média. Freitas et al. (2002) concluíram em seu trabalho que ALT seria o caráter mais promissor para seleção, porém DMC e FOR seriam caracteres pouco interessantes naquele estágio de desenvolvimento de M. urundeuva para efetivar um estudo de seleção. Sebbenn e Ettori (2001) obtiveram resultados altos para esse quociente e para o caráter altura, para as três espécies em cultivo misto. Entre as espécies estudadas neste trabalho, os autores observam que $M$. urundeuva apresentou a maior razão $(0,92)$. Sebbenn et al. (1999) obtiveram razões mais altas para altura total de planta em Pterogyne nitens $(0,65)$.

A expressão da variação entre plantas dentro de progênies, determinada pelo coeficiente de variação fenotípico dentro de progênies $\left(\mathrm{CV}_{\mathrm{D}}\right)$, apresentou valor médio de $30,3 \%$ para o terceiro ano após o plantio, sendo o menor valor de $6,8 \%$ para o caráter D30 e o maior de $69,3 \%$ para FOR. No quarto ano, o coeficiente de variação fenotípico dentro de progênies apresentou valor médio de $26,3 \%$, sendo o menor de $16,4 \%$ para o caráter ALT e o maior valor de 45,2\% para FOR, sendo que, nesse ano, os dados se apresentaram mais homogêneos que no terceiro ano de coleta, para todos os caracteres avaliados.

O coeficiente de variação fenotípico dentro de progênies $\left(\mathrm{CV}_{\mathrm{D}}\right)$, entre todos os caracteres avaliados, apresentou-se superior ao coeficiente de variação genética, supondo que o material é de grande potencial genético para a seleção dentro de famílias. Sebbenn et al. (1999) observaram resultado semelhante, estudando populações de $P$. nitens; citaram que 3/4 da variância genética aditiva se encontrava entre plantas dentro de famílias. Esses autores observaram que esse parâmetro é de grande importância em programas de melhoramento, dado que permite um ganho adicional, pela seleção dentro de progênies, e a magnitude dos valores do coeficiente de variação fenotípica dentro de progênies reforça a hipótese da eficiência de ganhos em trabalhos de melhoramento com a espécie. 


\section{Herdabilidades}

A utilização do coeficiente de correlação intraclasse $\left(r_{x y}=0,37\right)$, estimado da taxa de cruzamento por Freitas (2003), causou redução substancial na estimativa da variância genética aditiva, em todos os caracteres, nos três experimentos, nos dois anos de coleta de dados. A redução foi de $32,8 \%$ em relação ao modelo aleatório (meios-irmãos). Portanto, a estimativa dos coeficientes de herdabilidade e os ganhos esperados na seleção estariam superestimados nesse valor, caso o modelo de cruzamentos aleatórios fosse utilizado.

Os valores obtidos para herdabilidade, nos diferentes níveis, evidenciam que resultados superestimados podem levar a uma quantificação errônea da herança dos caracteres, induzindo a erros nas estimativas de variâncias genéticas aditivas e herdabilidades e, consequientemente, nas estimativas de ganhos genéticos. Esses dados apontam que a seleção, nesse caso, deve-se basear na herdabilidade estimada para o sistema misto. Resultados semelhantes foram observados por Sebbenn et al. (2000), em Cariniana legalis (Mart.) O. Ktze. Em termos gerais, esses resultados reforçam a importância do conhecimento do sistema de reprodução, quando se pretendem estimar os parâmetros genéticos para fins de seleção. A semelhança entre os coeficientes de herdabilidade em nível de plantas e herdabilidade em nível de plantas, dentro de parcelas, ocorre, pois praticamente medem o mesmo efeito: a herança dos caracteres em nível de plantas individuais e a média individual dentro de progênies (SEBBENN e ETTORI, 2001). Esses dados evidenciam que a seleção, nesse caso, deve-se basear principalmente na herdabilidade, em nível de média de progênies, em que o controle da variação fenotípica tem maior efeito genético, permitindo maior sucesso na seleção.

O coeficiente de herdabilidade, no sentido restrito, em nível de plantas $\left(\hat{h}^{2}\right)$, nos dois anos de coleta de dados, foi baixo, variando de 0,001 a 0,59. O mesmo se pode observar na herdabilidade, em nível de plantas, dentro de parcelas $\left(\hat{h}_{D}^{2}\right)$, com valores variando entre 0,001 e 0,64. A herdabilidade, em nível de média de parcelas $\left(\hat{h}_{x}^{2}\right)$, apresentou valores entre 0,001 e 0,61.

Verifica-se, respectivamente ao terceiro e quarto anos após o plantio (Tabela 6), que a herdabilidade, em nível de média de parcelas, foi superior, para a maioria dos caracteres, aos valores apresentados para a herdabilidade, em nível de plantas, dentro de parcelas. A herdabilidade, no sentido restrito, em nível de plantas, apresentou valores superiores aos da herdabilidade, em nível de plantas, no sistema misto de cruzamento, o mesmo ocorrendo para a herdabilidade, em nível de plantas, dentro de parcelas.

Entre os experimentos, observa-se que o cultivo homogêneo (ARO) apresentou os menores índices de herdabilidade em praticamente todos os níveis.

Valores baixos para herdabilidade, em nível de plantas, foram observados por diversos autores. Freitas et al. (2002) obtiveram média baixa, inferior à que foi encontrada neste trabalho $(0,06)$, indicando que o controle genético é fraco, nesse nível, assim como os que foram obtidos por Moraes et al. (1992), em duas populações de $M$. urundeuva, com 0,08 e 0,07, respectivamente. Sebbenn et al. (2000), em três populações de Cariniana legalis, obtiveram baixas estimativas para a herdabilidade, em nível de planta $(0,12)$. Entretanto, Sebbenn e Ettori (2001) obtiveram, para M. urundeuva, herdabilidade, em nível de planta de 0,64 e 0,48, respectivamente, para diâmetro à altura do peito e altura total da planta. A herdabilidade, em nível de média de progênies, em $M$. urundeuva, foi avaliada por Oliveira et al. (2000) durante o período de 7 e 24 meses de idade. Esses autores constataram que, no plantio heterogêneo, os valores foram de médio para alto, variando entre 0,12 e 0,50, com média de 0,33 , semelhante aos valores obtidos neste trabalho. No plantio homogêneo, os valores foram muito baixos, com média de 0,08 e valores variando entre zero e 0,44 . Pode-se inferir, portanto, que os plantios mistos são mais favoráveis à detecção de efeitos genéticos.

\section{CONCLUSÕES}

O estudo da variação genética em progênies de $M$. urundeuva resultou nas seguintes conclusões:

a) Existe variação genética entre progênies no plantio puro.

b) Os maiores crescimentos foram observados no consórcio aroeira, mutambo e angico (AMA).

c) M. urundeuva plantada em sistema puro (ARO) apresenta melhor forma do fuste que plantios heterogêneos.

d) As progênies no sistema aroeira eucalipto (AEU) expressam maior variação genética, herdabilidade $\mathrm{e}$

R. Árvore, Viçosa-MG, v.30, n.3, p.319-329, 2006 
potencial para seleção de caracteres quantitativos do que nos sistemas AMA e ARO.

\section{AGRADECIMENTOS}

À FAPESP, pelo apoio financeiro; e aos técnicos Alexandre Marques da Silva, Ailton dos Reis e Manoel Fernando da Rocha Bonfim, pelo apoio na coleta dos dados.

\section{REFERÊNCIAS BIBLIOGRÁFICAS}

ALlARD, R.W. Princípios do melhoramento genético das plantas. São Paulo: Edgard Blücher, 1971. 381p.

EMPRESA BRASILEIRA DE PESQUISA AGROPECUÁRIA - EMBRAPA Sistema brasileiro de classificação de solos. Rio de Janeiro: EMBRAPA/CNPSO, 1999. 412p.

FAO. Databook on endagered tree and shrub species and provenances. Roma: 1986. p. 116-25.

FREITAS, M.L.M. Caracterização genética por marcador fAFLP e caracteres quantitativos em uma população de Myracrodruon urundeuva F.F. \& M.F. Allemão. 2003. 99f. Tese (Doutorado em Genética e Melhoramento de Plantas) Universidade Estadual Paulista, Jaboticabal, 2003.

FREITAS, M.L.M.; MORAES, M.L.T.; BUZETTI, S. Variação genética em progênies de Myracrodruon urundeuva Fr. All. em diferentes sistemas de plantio. R. Inst. Flor., v. 14, n. 2, p. 133-141, 2002.

HAMRICK, J.L. The distribution of genetic variation within and among natural plant populations. In: SCHONEWALD-COX, C.M. et al. (Ed.). Genetics and conservation. Menlo Park: Benjamin Cummings Publishing Company, 1983. p. 335-348.

KAGEYAMA, P.Y. Genetic structure of tropical tree species of Brazil. In: BAWA, K.S.; HADLEY, M. (Eds.). Reproductive ecology of tropical forest plants. Paris: UNESCO, 1990.

KAGEYAMA, P.Y. Variação genética em progênies de uma população de Eucalyptus grandis Hill ex. Maiden. 1980. 125f. Tese (Doutorado em Genética e Melhoramento de Plantas) - Escola Superior de Agricultura “Luiz de Queiroz”, Piracicaba, 1980.

R. Árvore, Viçosa-MG, v.30, n.3, p.319-329, 2006
KAGEYAMA, P.Y.; DIAS, I.S. Aplicación de conceptos genéticos a espécies forestales nativas en Brasil. Información sobre Recursos Genéticos Forestales, v. 13, p. 2-10. 1985.

MORAES, M.L.T. et al. Variação genética em duas populações de aroeira (Astronium urundeuva-(Fr. All). Engl-Anacardiaceae). R. Inst. Flor., v. 1, n. 1, p. 1241-1245, 1992.

OLIVEIRA, S.A. et al. Variação genética em progênies de aroeira (Myracrodruon urundeuva Fr. All.) sob diferentes condições de cultivo. I Aspectos silviculturais. R. Inst. Flor., v. 12, n. 2, p. $155-166.2000$.

PAIVA, J.R.; VALOIS, A.C.C. Espécies selvagens e sua utilização no melhoramento. In: NASS, L. et al. (Eds.) Recursos genéticos e melhoramento - plantas. Rondonópolis: Fundação MT, 2001. p. 79-99.

PIMENTEL-GOMES, F.; GARCIA, C.H. Estatística aplicada a experimentos agronômicos e florestais: exposição com exemplos e orientações para uso de aplicativos. Piracicaba: FEALQ, 2002. 309p.

RITLAND, K. Correlated matings in the partial selfer Mimulus guttatus. Evolution, v. 43 , p. 848-859, 1989.

S.A.S. Institute. SAS procedures guide. Version 8 (TSMO). Cary: 1999. 2v.

SEBBENN, A.M.; ETTORI, L.C. Conservação genética ex situ de Esenbeckia leiocarpa, Myracrodruon urundeuva e Peltrophorum dubium em teste de progênies misto. R. Inst. Flor., v. 13, n. 22, p. 201-211, 2001.

SEBBENN, A.M. et al. Taxa de cruzamento em populações de Cariniana legalis (Mart.) O. Ktze.: Implicações para a conservação e o melhoramento genético. Sci. For., v. 58, p. 25-40, 2000.

SEBBENN, A.M. et al. Variação genética entre e dentro de populações de amendoim - Pterogyne nitens. Sci. For., v. 56, p. 29-40, 1999. 
SEBBENN, A.M.; KAGEYAMA, P.Y.;

VENCOSVKY, R. Variabilidade genética, sistema reprodutivo e estrutura genética espacial em Genipa americana L. através de marcadores isoenzimáticos. Sci. For., v. 53, p. 15-30. 1998.

SIQUEIRA, A.C.M.F. et al. Variação genética entre e dentro de populações de

Balfourodendron riedelianum (Engler) para conservação ex situ. R. Inst. Flor., v. 12, n. 2, p. 89-103. 2000.

SIQUEIRA, A.C.M.F.; NOGUEIRA, J.C.B.;

KAGEYAMA, P.Y. Conservação dos recursos genéticos ex situ do Cumbaru (Dipteryx alata) Vog. - Leguminosae. R. Inst. Flor., São Paulo, v. 5, n. 2, p. 231-243. 1993.
STRANGHETTI, V. Levantamento florístico das espécies vasculares de uma floresta estacional no Norte do Estado de São Paulo, Estação Ecológica de Paulo de Faria. 1996. 164f. Tese (Doutorado em Ciências Biológicas) - Universidade Estadual de Campinas, Campinas, 1996.

VENCOVSKY, R. Herança quantitativa. In: PATERNIANI, E. (Ed.) Melhoramento e produção de milho. Piracicaba: Fundação Cargill, 1987. p. 137-214.

VENCOVSKY, R.; BARRIGA, P. Genética biométrica no fitomelhoramento. Ribeirão Preto: Sociedade Brasileira de Genética, 1992. 496p. 